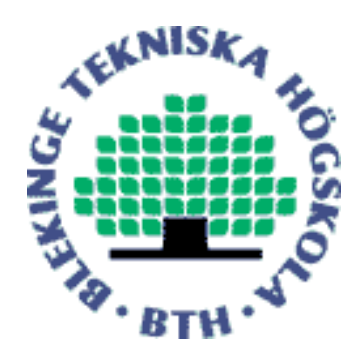

Electronic Research Archive of Blekinge Institute of Technology http://www.bth.se/fou/

This is an author produced version of a paper published in World Scientific/ Computational Intelligence in Decision and Control FLINS 2008, 21-24 September, 2008, Madrid. This paper has been peer-reviewed but may not include the final publisher proofcorrections or journal pagination.

Citation for the published paper:

Elisabeth Rakus-Andersson, Maria Salomonsson, Hang Zettervall. Two-player Games with Fuzzy Entries of the Payoff Matrix. World Scientific, 2008, Vol. 1, pp. 593-598/ Computational Intelligence in Decision and Control - FLINS 2008, 21-24 September, 2008, Madrid. 


\title{
TWO-PLAYER GAMES WITH FUZZY ENTRIES OF THE PAYOFF MATRIX
}

\author{
ELISABETH RAKUS-ANDERSSON \\ Blekinge Institute of Technology, Department of Mathematics and Science, \\ S-37179 Karlskrona, Sweden \\ MARIA SALOMONSSON \\ Blekinge Institute of Technology, Department of Mathematics and Science, \\ S-37179 Karlskrona, Sweden \\ HANG ZETTERVALL \\ Blekinge Institute of Technology, Department of Mathematics and Science, \\ S-37179 Karlskrona, Sweden
}

\begin{abstract}
We expand the classical model of a two-player game by inserting of fuzzy sets as payoff values in the game matrix. Players can thus formulate their payoff expectations with words instead of deciding on numerical entries of the matrix. In this way we count on the better verbal communication between players when designing the preliminaries of the game. As a final result we expect to obtain samples of the players' optimal strategies, which will preserve the profit of the game on the neutral level.
\end{abstract}

\section{Introduction}

Nowadays, a large number of contributions to fuzzy games are found in literature, e.g., in $[3,4,5,6]$. The majority of models are based on the classical crisp method of maintaining of the equilibrium [1, 2], provided that assumptions of fuzzy approaches differ a lot from each other.

We sketch an original simple counterpart of the two-player game with imprecise coefficients of the payoff matrix. The execution of the algorithm provides us with a sample of optimal strategies selected from all tactics prepared by the players, on condition that an objective of the game is to keep the balance status of a profit variable. We thus reject the strategies causing extreme deviations from the null level. Moreover, we can appreciate the directions of deviations from the balance caused by the strategies recognized as optimal.

In Section 2 we discuss a theoretical aspect of the proposed method to practise its action on medical and technical examples in Sections 3 and 4. 


\section{The Model of a Two-player Game with Imprecise Payoff Values}

Assume the existence of two players $A$ and $B$ who adopt their action plans called strategies to win a game. The row player $A$ uses his strategies $R_{r}, r=1, \ldots, m$, whereas the column player $B$ makes a selection among his strategies $K_{k}, k=1$, $\ldots, n$. Suppose further that $A$ and $B$ choose the strategies independently of each other, i.e., we emphasize that none of them has knowledge about the opponent's tactics of replying. Both $A$ and $B$ select their strategies $R_{r}$ and $K_{k}$ respectively, which results in obtaining a gain by one of them and a loss by the other. The gains and the losses are preliminarily determined for each pair of strategies $\left(R_{r}\right.$, $\left.K_{k}\right)$ due to a profit plan yielded by a payoff matrix $P=\left\{\left(\left(R_{r}, K_{k}\right), \mu_{P}\left(R_{r}, K_{k}\right)\right)\right\}$, where all $\mu_{P}\left(R_{r}, K_{k}\right)$ represent fuzzy sets attached to verbal descriptions of the profit, $r=1, \ldots, m, k=1, \ldots, n$.

By "profit" we mean the changes of a certain variable in either positive or negative directions. We wish to preserve the final profit of the game on a balance level near zero after sampling of the most accurate strategies for that purpose. $A$ thus aims at finding the strategy that, used against $B$ 's strategies, allows $A$ to keep his gain near the zero balance level. $B$ intends to test his strategies contra $A$ 's answers to select one providing him with a loss near zero.

The players wish to determine the matrix $P$ as a collection of verbal expressions at the initial stage of the game. Let us then differentiate changes in the profit variable status as terms of a linguistic variable "changes of the profit variable" = $\{$ "nb" = "negatively big”, " $n s "=$ "negatively small”, " $z$ " = "near zero", "ps” = "positively small”, " $p b$ ” = "positively big” $\}$. Each notion should be assimilated with a corresponding fuzzy set that constitutes the primary entry of $P$ intended for the pair $\left(R_{r}, K_{k}\right)$ to be finally translated in $\mu_{P}\left(R_{r}, K_{k}\right) \in[0,1]$.

All $\mu_{P}\left(R_{r}, K_{k}\right)$ denote the gains of $A$ related to $B$ 's losses on condition that larger $\mu_{P}\left(R_{r}, K_{k}\right)$ correspond to positively directed changes of the profit variable for $A$, whereas lower $\mu_{P}\left(R_{r}, K_{k}\right)$ in $A$ 's case are associated with negative deviations of the variable status. The values of $\mu_{P}\left(R_{r}, K_{k}\right)$ are interpreted in the opposite directions for $B$. The presence of $\mu_{P}\left(R_{r}, K_{k}\right)=0.5$ in the matrix emphasizes the existence of the profit on the zero level when using $R_{r}$ against $K_{k}$. We design fuzzy sets attached to the levels over the interval of profit changes $\left[a_{\min }<0, a_{\max }>0\right]$, adjusted to a type of the profit variable. We determine the sets by laying of the following restrictions over $\left[a_{\min }, a_{\max }\right]$ :

$$
\mu_{n n b^{n}}(x)=\left\{\begin{array}{lll}
1 & \text { for } & x<a_{\min }, \\
\frac{4}{a_{\min }} x-3 & \text { for } & a_{\min } \leq x \leq \frac{3}{4} a_{\min }, \\
0 & \text { for } & x>\frac{3}{4} a_{\min },
\end{array}\right.
$$




$$
\begin{gathered}
\mu_{" n s "}(x)=\left\{\begin{array}{llc}
1 & \text { for } & x<\frac{1}{2} a_{\min }, \\
\frac{4}{a_{\min }} x-1 & \text { for } & \frac{1}{2} a_{\min } \leq x \leq \frac{1}{4} a_{\min }, \\
0 & \text { for } & x>\frac{1}{4} a_{\min },
\end{array}\right. \\
\mu_{" z^{\prime \prime}}(x)=\left\{\begin{array}{ccc}
0 & \text { for } & x<\frac{1}{2} a_{\min }, \\
\frac{-2}{a_{\min }} x+1 & \text { for } & \frac{1}{2} a_{\min } \leq x \leq 0, \\
\frac{-2}{a_{\max }} x+1 & \text { for } & 0 \leq x \leq \frac{1}{2} a_{\max }, \\
0 & \text { for } & x>\frac{1}{2} a_{\max },
\end{array}\right. \\
\mu_{" p s "}(x)=\left\{\begin{array}{ccc}
0 & \text { for } & x<\frac{1}{4} a_{\max }, \\
\frac{4}{a_{\max }} x-1 & \text { for } & \frac{1}{4} a_{\max } \leq x \leq \frac{1}{2} a_{\max }, \\
1 & \text { for } & x>\frac{1}{2} a_{\max },
\end{array}\right.
\end{gathered}
$$

and

$$
\mu_{" p b "}(x)=\left\{\begin{array}{lll}
0 & \text { for } & x<\frac{3}{4} a_{\max }, \\
\frac{4}{a_{\max }} x-3 & \text { for } & \frac{3}{4} a_{\max } \leq x \leq a_{\max } \\
1 & \text { for } & x>a_{\max } .
\end{array}\right.
$$

In Fig. 1 we plot all membership functions of the fuzzy sets representing the members of the list "changes of the profit variable".

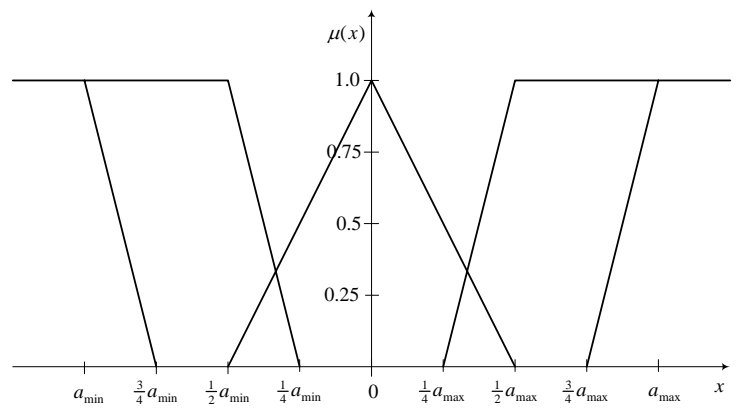

Figure 1. Fuzzy sets representing the terms of "changes of the profit variable"

In order to design the payoff matrix in the numerical shape, we extract one value of each set support as an indicator of the set. We accept such element $x$ of 
the set support, which is assimilated to a certain threshold of the set's membership function. We decide to state the $x$ value as the $x$-coordinate of an intersection point between the line $\mu_{\text {set }}(x)=1$ and a part of the membership function in which $\mu_{\text {set }}(x)<1$.

The expressions coming from "changes of the profit variable" have thus the representatives decided as $x_{" n b "}=a_{\min }, x_{n n s "}=\frac{1}{2} a_{\min }, x_{" z^{\prime}}=0, x_{n} p s^{\prime \prime}=\frac{1}{2} a_{\max }$ and $x_{" p b "}=a_{\max }$. If we construct another fuzzy set "change” over $\left[a_{\min }, a_{\max }\right]$ impacted by a membership function

$$
\mu_{\text {"change" }}(x)= \begin{cases}0 & \text { for } x<a_{\min }, \\ \frac{1}{2}\left(-\frac{x}{a_{\min }}+1\right) & \text { for } a_{\min } \leq x \leq 0, \\ \frac{1}{2}\left(\frac{x}{a_{\max }}+1\right) & \text { for } 0 \leq x \leq a_{\max }, \\ 1 & \text { for } x>a_{\max },\end{cases}
$$

then we will get $\mu_{\text {"change" }}\left(a_{\text {min }}\right)=0, \mu_{\text {"change" }}\left(\frac{1}{2} a_{\text {min }}\right)=0.25, \mu_{\text {"change" }}(0)=0.5$, $\mu_{\text {"change" }}\left(\frac{1}{2} a_{\text {max }}\right)=0.75$ and $\mu_{\text {"change" }}\left(a_{\max }\right)=1$ as the sets' final replacements.

In matrix $P$ the entries of $\left(R_{r}, K_{k}\right)$ are first determined by the sets: “ $n b$ ", " $n s$ ", " $z$ ", " $p s$ " or " $p b$ " to be then replaced by $\mu_{P}\left(R_{r}, K_{k}\right)$, being one of the values: $0,0.25,0.5,0.75$ or 1 . We can always extend the number of notions in the list in conformity with our needs.

Having obtained the numerical data in $P$ we make two judgments, namely, a selection of the best strategies for $A$ and $B$ in order to keep the profit invariable near zero. A maximizes minimal gains predicted one by one for the strategies $R_{r}$ provided that $B$ replies to $R_{r}$ with one strategy among $K_{1}, \ldots, K_{n}$, whose choice is unknown for $A$. The selection of the optimal strategy $R^{*}$ of $A$ is supported by a value $\mu_{A}\left(R^{*}\right)$ introduced by a formula

$$
\mu_{A}\left(R^{*}\right)=\max _{1 \leq r \leq m}\left(\min \left(\mu_{P}\left(R_{r}, K_{1}\right), \ldots, \mu_{P}\left(R_{r}, K_{n}\right)\right) .\right.
$$

If the value of $\mu_{A}\left(R^{*}\right)$ appears in row $r$ then strategy $R_{r}$, marking the row $r$, will be considered as the optimal strategy $R^{*}=R_{r}, r=1, \ldots, m$.

Since $B$ minimizes his largest losses then we should seek the optimal $K^{*}$ strategy used against an unknown choice of $R_{1}, \ldots, R_{m}$ to maintain the profit around zero. We compute $\mu_{B}\left(K^{*}\right)$, which helps to indicate $K^{*}$, as

$$
\mu_{B}\left(K^{*}\right)=\min _{1 \leq k \leq n}\left(\max \left(\mu_{P}\left(R_{1}, K_{k}\right), \ldots, \mu_{P}\left(R_{m}, K_{k}\right)\right) .\right.
$$

Strategy $K^{*}=K_{k}$ will be accepted as $B$ 's optimal method used in the game if the value of $\mu_{B}\left(K^{*}\right)$ is found in column $k, k=1, \ldots, n$. 
After having found $R^{*}$ and $K^{*}$ (there can be more than one optimal strategy belonging to the player) we reject the techniques leading to extreme values of the profit variable. For the optimal strategies we can classify sizes of deviations from the zero level via studying of the values of $\mu_{A}\left(R^{*}\right)$ and $\mu_{B}\left(K^{*}\right)$.

\section{Optimal Strategies in Maintaining of Body Weight}

A patient with the diabetes-type II has to keep weight changes near zero. The changes are expected to happen in $\left[a_{\min }, a_{\max }\right]=[-4 \mathrm{~kg}, 4 \mathrm{~kg}]$. Two teams $A$ and $B$ consist of physicians, dieticians and gym-specialists. A prepares strategies $R_{1}$, $R_{2}, R_{3}$ contra $B$ 's responses $K_{1}, K_{2}, K_{3}$ to help the patient in curing of the illness and in preserving of his body weight at the unchangeable level, which can be contradictory. Both $A$ and $B$ are aware of effects of their joined-in-pair strategies, initially estimated as changes of weight in the following matrices:

$$
\begin{aligned}
& \begin{array}{llllll}
K_{1} & K_{2} & K_{3} & K_{1} & K_{2} & K_{3}
\end{array}
\end{aligned}
$$

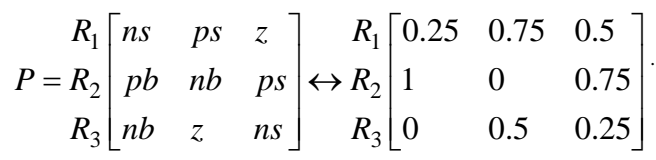

For $A$, due to (7), $\mu_{A}\left(R^{*}\right)=\max (\min (0.25,0.75,0.5), \min (1,0,0.75)$, $\min (0,0.5,0.25))=0.25$. $A$ should thus choose $R_{1}$ and $R_{3}$ against the unknown selection of $B$ 's tactics with a risk to cause a small negative change of the patient's weight. To state a sample of optimal strategies for $B$ we evaluate $\mu_{B}\left(K^{*}\right)=\min (\max (0.25,1,0), \max (0.75,0,0.5), \max (0.5,0.75,0.25))=0,75$. $B$ should use strategies $K_{2}$ and $K_{3}$ that are expected to keep the patient's body weight at the "negative small” level. $R_{2}$ and $K_{1}$ are rejected as methods leading to too large changes of the weight.

\section{The Constant Radio Transmission Rate in a Fuzzy Game}

The radio spectrum is a limited and regulated resource. By using more channels, more data can be transmitted in the same period of time. However, if an overlap occurs in the channels picked, i.e., if several transmissions are attempted on a channel, transmissions can still take place but less data is transmitted due to the overlap. The amount of data that can be transmitted by using overlapped channel(s) depends on several factors. The aim is to keep the data rate constant and equally divided amongst the players. In our example, we assume that the frequency spectrum is divided into three channels. Players $A$ and $B$ choose 
among three different channels (and may choose more than one channel) independently of each other. $A$ uses the strategies $R_{1}, R_{2}, R_{3}$, and $B$ the strategies $K_{1}, K_{2}, K_{3}$. All strategies refer to a specific channel selection configuration. The changes are expected to happen within $\left[a_{\min }, a_{\max }\right]=[-3 \mathrm{Mbps}, 3 \mathrm{Mbps}]$. An illustrative payoff matrix can thus be formulated as:

$$
\begin{aligned}
& \begin{array}{lll}
K_{1} & K_{2} & K_{3}
\end{array} \\
& P=R_{2} R_{1}\left[\begin{array}{lll}
0.5 & 0.5 & 0.75 \\
1 & 0.75 & 0.25 \\
0 & 0.25 & 0.75
\end{array}\right]
\end{aligned}
$$

Hence, $A$ 's optimal gain is $\max (0.5,0.25,0)=0.5$ whereas $B$ reaches the optimal loss $\min (1,0.75,0.75)=0.75$. A payoff value larger than 0.5 , will give player $A$ an advantage in the sense of having access to higher transmission rates. The result of the example in this case indicates that $A$ 's influence of the strategies will lead to a constant transmission rate, while $B$ 's influence of the strategies will lead to a transmission rate change to the advantage of $A$. $A$ excludes $R_{2}$ and $R_{3}$, and $B$ does not use $K_{1}$ anymore.

\section{Conclusions}

The attempt of achieving of a balance level in the two-player game, supported by a fuzzy payoff matrix, results in composing of a method that provides two players with the best techniques. These strategies, applied to "profit variables", cause the least deviations of the variable values from the null status. We can even define directions of the deviations in the verbal forms by returning to the association rules between the terms of "changes of the profit variable" and the values of $\mu_{A}\left(R^{*}\right)$ or $\mu_{B}\left(K^{*}\right)$.

\section{References}

1. J. Nash, Proc. Nat. Academy Sci., 36, 48 (1950).

2. L. Shapley, Contributions to the Theory of Games. Princeton, NJ: Princeton Univ. Press, 207 (1953).

3. D. Butnariu, Fuzzy Sets and Syst., 1, 181 (1978).

4. P. Borges, R. Pacheco, R. Barcia and S. Khator, BioSystems 41, 127, (1997).

5. Yan-An Hwang, Fuzzy Sets and Systems, 158, 2480 (2007).

6. F. Kacher and M. Larbani, Fuzzy Sets and Systems, 159, 164 (2008). 